\title{
Prevalence of Psychiatric Disorders Among Children of Different Ethnic Origin
}

\author{
Barbara W. C. Zwirs • Huibert Burger • \\ Tom W. J. Schulpen • Martin Wiznitzer • Hans Fedder • \\ Jan K. Buitelaar
}

Published online: 7 March 2007

(C) Springer Science + Business Media, LLC 2007

\begin{abstract}
The present study assesses the population prevalence of DSM-IV disorders among native and immigrant children living in low socio-economic status (SES) innercity neighborhoods in the Netherlands. In the first phase of a two-phase epidemiological design, teachers screened an ethnically diverse sample of 2041 children aged 6-10 years
\end{abstract}

B. W. C. Zwirs - T. W. J. Schulpen

Department of Paediatrics, University Medical Centre Utrecht, Utrecht, The Netherlands

B. W. C. Zwirs $(\bowtie)$

School of Law, Erasmus University Rotterdam,

Burg. Oudlaan 50, room L6-34, P.O. Box 1738, NL-3000 DR,

Rotterdam, The Netherlands

e-mail: Zwirs@frg.eur.nl

H. Burger

Julius Centre for Health Sciences and Primary Care,

University Medical Centre Utrecht,

Utrecht, The Netherlands

H. Burger

Department of Epidemiology and Department of Psychiatry, University Medical Centre Groningen,

Groningen

M. Wiznitzer

Justice Department, Netherlands Institute of Forensic

Psychiatry and Psychology,

Amsterdam, The Netherlands

H. Fedder

Ambulatory Care Institutions,

Middelburg

J. K. Buitelaar

Department of Psychiatry, and Academic Centre for Child and

Adolescent Psychiatry, Radboud University Nijmegen

Medical Centre,

Nijmegen, The Netherlands using the Strengths and Difficulties Questionnaire (SDQ). In the second phase, a subsample of 253 children was psychiatrically examined, while their parents were interviewed. In addition, teachers completed a short questionnaire about 10 DSM-IV items. Prevalence was estimated using the bestestimate diagnosis based on parent, child and teacher information. Projected to the total population, $11 \%$ of the children had one or more impairing psychiatric disorders, which did not differ between native and non-native children. In the total group a clear relationship was observed between the prevalence of psychiatric disorders and gender, parental psychopathology, peer problems and school problems, but not among all ethnic groups separately. This study suggests that the prevalence of psychiatric disorders among non-treated minority and native children in low SES inner-city neighborhoods does not materially differ. However, associated mechanisms may be influenced by ethnicity.

Keywords Prevalence · Psychiatric disorders · Ethnicity

In the design of preventive interventions and treatment programs for youth, policy makers need to know the prevalence of psychiatric disorders among children. With the increasing ethnic diversity of populations worldwide, comparing the prevalence of psychiatric disorders in children of different ethnic background is of particular interest. Further, differences in occurrence in psychiatric disorders between ethnicities may yield clues to their etiology. More specifically, when the prevalence of psychiatric disorders may be lower or higher among a particular ethnic group, ethnically specific factors may explain this difference.

The majority of epidemiological studies on childhood psychopathology have been carried out in the United States (US). Relatively few studies have been conducted in Europe, Asia, Africa, and South America. In a review of these studies, 
Roberts reports prevalence estimates of child psychopathology ranging from $1 \%$ in Tokyo to $51 \%$ in the US (Roberts, Attkisson, \& Rosenblatt, 1998). Certainly, methodological variations across studies, such as sampling differences, have contributed to this wide range of prevalence estimates. However, the precise effect of these method factors is difficult to judge. Hence, it remains unclear to what extent the observed differences in prevalence are due to perceptual bias or reflect actual behavior differences.

Therefore, it is important to conduct epidemiological studies on the prevalence of childhood disorders in different ethnic groups from one population and with one single methodology. For instance, Costello (1997) compared American Indian and White youth and reported that the overall prevalence of psychiatric disorders did not vary according to ethnicity (Costello, Farmer, Angold, Burns, \& Erkanli, 1997). Similarly, no ethnic difference was observed in the overall prevalence of psychiatric disorders between African American and white youth (Angold et al., 2002). In Continental Europe, only three studies on the prevalence of problem behavior in children compared minority with majority ethnic groups, i.e. Turkish immigrant adolescents with native Dutch adolescents (Bengi-Arslan, Verhulst, Van der Ende, \& Erol, 1997; Crijnen, Bengi-Arslan, \& Verhulst, 2000; Murad, Joung, van Lenthe, Bengi-Arslan, \& Crijnen, 2003), indigenous Sami with majority Norwegian adolescents (Kvernmo \& Heyerdahl, 1998) and Moroccan immigrant youth with native Dutch youth (Stevens et al., 2003). However, these European studies were based on rating scales. Thus, studies that estimate the prevalence of child psychiatric disorders in different ethnic groups from one population using structured and clinical interviews are scarce and have not been performed in Europe.

The present study was designed to extend the knowledge in this area by assessing the population prevalence of psychiatric disorders among non-treated children of the four largest ethnic groups in the Netherlands, namely Dutch, Moroccan, Turkish and Surinamese, using the best-estimate procedure. In the 1960 and early 1970s Turks and Moroccans migrated from Mediterranean countries to the Netherlands as lowwage labor migrants, whereas Surinamese have come from South America to the Netherlands during the process of decolonization since 1975. As the different ethnic groups were from one population and as we used one single methodology, the prevalence estimates in non-Dutch immigrant children were directly comparable to those in native Dutch children.

Because immigrants may be confronted with stress causing factors that may threaten psychological adjustment, such as discrimination by the host country and structural deprivation, we expect the prevalence of psychiatric disorders to be higher for the non-Dutch children. Moreover, as the cultural gap is probably the largest for the Moroccans and the smallest for the Surinamese, we expect the prevalence of psychiatric disorders among the non-Dutch groups to be the highest for the Moroccan and the lowest for the Surinamese children. Previous studies yielded inconsistent findings concerning ethnic differences in the prevalence of psychiatric disorders between Moroccan immigrant and Dutch native adolescents (Stevens et al., 2003) and between Turkish immigrant and Dutch native children (Crijnen et al., 2000; Murad et al., 2003). However, as these studies were based on questionnaires and missing a gold standard the reported differences may be explained by perceptual differences rather than by actual differences in problem behavior. To our knowledge, no studies exist on the prevalence of psychiatric disorders among Surinamese children.

The second aim of the study was to examine associated mechanisms of psychiatric disorders across the four ethnic groups. We assessed demographic variables, such as gender, age and socio-economic status (SES), and psychosocial variables, such as parental psychopathology, school problems, peer problems and country of birth. Based on the literature we expected a negative association with SES (Verhulst \& Achenbach, 1995b), but a positive association with parental psychopathology (Johnson, Cohen, Kasen, \& Brook, 2006), peer problems and school problems (Sowa, Crijnen, BengiArslan, \& Verhulst, 2000). In addition, we expected psychiatric disorders to be more prevalent among boys than among girls. We had no reason to expect a strong ethnic effect on these associations.

\section{Methods}

\section{Subjects}

The study sample used in the present study is part of a larger study on the detection of ADHD among children of Different Ethnic Origins in the Netherlands (ADEON-study). The ADEON-study was designed to estimate the prevalence of behavioral disorders in non-treated native Dutch, Moroccan, Turkish and Surinamese children living in the Netherlands. In order to screen a large sample, while restricting the number of extensive diagnostic interviews, a two-stage design was used (Armitage, Berry, \& Matthews, 1987).

In the first phase of the ADEON-study (Zwirs, Burger, Schulpen, \& Buitelaar, 2006), parents of 2802 children, enrolled in grade 3 through 5 of mainstream schools were asked permission for their child's teacher to be administered the Strengths and Difficulties Questionnaire (SDQ) (Goodman, 1997). To obtain a sample with all four ethnicities represented with similar SES, we sampled exclusively from low SES inner-city areas in two large cities in the Netherlands (i.e. Amsterdam and Utrecht), as most migrants live in low SES urban neighborhoods. The SES level of neighborhoods has previously been determined according to the unemployment rate, the level of education and income (Knol, 1998). 
Forty-five (52\%) of the 87 eligible schools, participated in the study. Of 37 schools $(82 \%)$ all teachers participated, the majority being of Dutch origin (77\%). The reasons for non-participation among schools and teachers were mainly logistic, i.e. they already participated in other studies or they were too occupied with other activities. As both participating and non-participating schools are from the same neighborhoods, we assume the socio-demographic characteristics of the children in the 45 participating schools to be similar to those in the 42 schools that did not participate. Therefore, we consider selection bias unlikely. Parents of all 2802 children in these three grades received a letter with information on the screening procedures.

Children with another ethnic origin than Dutch, Moroccan, Turkish or Surinamese, were excluded from the study $(N=336)$. According to the Dutch standard classification of ethnic groups (Dutch Central Bureau of Statistics), children were categorized as "Moroccan", "Turkish" or "Surinamese" when the child itself and/or at least one parent had been born in Morocco, Turkey or Surinam, respectively. When both parents were of non-Dutch origin, we used mother's country of birth to determine the child's ethnicity. Children were classified as "Dutch" when both parents and the child itself were born in the Netherlands. As 281 parents refused to give permission, 2185 (89\%) out of the remaining 2466 questionnaires were completed, with no significant variation in this proportion across ethnicity. After excluding 40 children with incomplete data and 104 children that were treated for behavioral problems or other psychiatric disorders, the study consisted of 2041 participants.

For the second phase, we invited all children scoring above the 90th percentile on problem behavior on the SDQ, denoted as screen-positives, and a random sample of children scoring below the 90th percentile on problem behavior, referred to as screen-negatives. We selected a random sample of the screen-negatives by assigning a computer generated random number to these children and then selecting those below a certain predefined cut-off. As a result, 376 children were eligible for participation in our study of whom 153 were screen-positive and 223 were screen-negative. Out of this group the parents of $270(72 \%)$ children participated, with no significant variation in this response rate between ethnic groups. Of the screen-positive children 99 (65\%) parents and of the screen-negative children 171 (77\%) parents participated. The analysis of the second phase was performed in all children with complete data $(N=253)$.

Data were collected from November 2002 to July 2004. Parent and child interviews took place at school in two separate rooms. When parents were not willing to attend the interview at school, they were visited at home. The child interviews were conducted by an experienced child psychiatrist and were all videotaped. Parents were interviewed by a trained psychologist or a trained medical student. When nec- essary, interviews with Turkish and Moroccan parents were conducted in Turkish, Moroccan Arabic, or Berber, respectively. After complete description of the study to the subjects, written informed consent was obtained from all parents. The study was approved by the Medical Ethical Committee of University Medical Centre Utrecht.

\section{Best-estimate diagnoses}

We used the best-estimate procedure for diagnosis: Results of the Semi-structured Clinical Interview for Children and Adolescents (SCICA) (McConaughy \& Achenbach, 2001) were considered in combination with the results of the outcomes of the Diagnostic Interview Schedule for Children-Parent Version (DISC-P) (Shaffer, Fisher, Lucas, Duldan, \& Schwab-Stone, 2000) and school-information. Best-estimate diagnoses were consensually formulated by a committee chaired by a board certificated psychiatrist (JB) and consisting of two other board certified child psychiatrists (HF and MW) who conducted the SCICA interviews as well as a psychologist/cultural anthropologist (BZ) who conducted DISC-P-interviews. A best-estimate diagnosis was attributed when this was indicated by at least two sources of information, i.e. parent-child, parent-teacher or teacher-child. In case of disagreement $(N=15)$, the child was recommended for the expert panel, consisting of the aforementioned professionals accompanied by two other psychiatrists, of whom one of non-Dutch origin, experienced in working with patients from non-Dutch ethnic origins. Additionally, 16 cases (4 children of each ethnic group) were assessed by this larger expert-panel to calibrate the best-estimate procedure. The large expert panel reviewed all available information and in addition viewed sections of the SCICA interview recorded on video. Best-estimate diagnoses were considered the reference diagnosis.

\section{Psychiatric interviews}

The Diagnostic Interview Schedule for Children-Parent Version (DISC-P) is a highly structured diagnostic interview and can be used by lay-persons to generate valid diagnoses (Shaffer et al., 2000) based on the Diagnostic and Statistical Manual of Mental Disorders-Fourth Edition (DSM-IV) (1994). The DISC, which was administered to the parents, is organized such that the different modules can be administered independently. We used the modules about Attention-deficit Hyperactivity Disorder (ADHD), Oppositional Defiant Disorder (ODD), Conduct Disorder (CD), Separation Anxiety Disorder (SAD), Generalized Anxiety Disorder (GAD), Posttraumatic Stress Disorder (PTSD), Major Depressive disorder (MDD) and Bipolar disorder (BD).

In a study by Schwab-Stone (1996) test-retest reliability has been found to be moderate for ADHD (kappa statistic 
$(\kappa)=0.60)$, ODD $(\kappa=0.68), \mathrm{CD}(\kappa=0.56)$ and $\mathrm{MDD}$ $(\kappa=0.55)$ and poor for $\operatorname{SAD}(\kappa=0.45)$. Agreement between the DISC and clinician ratings were moderate to very good except for SAD (Schwab-Stone et al., 1996). In the present study, the DISC's modules on externalizing disorders (i.e. ADHD, ODD and CD) yielded good levels of internal consistency across ethnicity with Cronbach's alpha coefficients of $0.85,0.83,0.73$ and 0.83 for the ADD scale, $0.78,0.81,0.66$ and 0.69 for the Hyperactivity-Impulsivity scale and 0.81 , $0.61,0.68$ and 0.69 for the ODD scale among the Dutch, Moroccan, Turkish and Surinamese groups, respectively. As most items on the other scales showed zero variance, it was not possible to calculate Cronbach's alpha's for these scales.

In addition, background information such as developmental history, family composition, country of birth, psychiatric disorders in the family and parental education were collected during the parent interview. Parental psychopathology was indicated when father and/or mother suffered from a psychiatric disorder. The socio-economic status (SES) was measured by parents' highest educational level which was rated on a 5-point scale: $0=$ no education; $1=$ elementary school; $2=$ secondary vocational; $3=$ secondary general; and $4=$ tertiary. SES was dichotomized in low SES (i.e. no education or elementary school) and high SES (i.e. secondary vocational, secondary general and tertiary).

The Semi-structured Clinical Interview for Children and Adolescents (SCICA), which was administered to the children, is a flexible semi-structured interview for assessing children aged 6-18 years (McConaughy et al., 2001). The good reliability and validity of the SCICA has been established for both an American sample (McConaughy et al., 2001) and a Dutch sample (Kasius, 1997). Furthermore, SCICA-items have found to be in line with DSM-IV diagnostic categories (McConaughy et al., 2001). The SCICA consists of nine broad areas of functioning 1. Activities, school, job; 2. Friends; 3. Family Relations; 4. Fantasies; 5. Self perception, Feelings; 6 . Parent/Teacher-Reported Problems; 7. Achievement Tests (optional); 8. For ages 6-11: Screen for Fine and Gross Motor Abnormalities (optional) and 9. For ages 12-18: Somatic Complaints, Alcohol, Drugs, Trouble with the Law (McConaughy et al., 2001).

As the SCICA asks about the child's perception of problems reported by parents or teachers, we administered a short questionnaire to teachers which was completed prior to the SCICA interview. This questionnaire included 10 DSM-IV items (American Psychiatric Association, 1994): 2 ADHD items on inattention, 2 ADHD items about hyperactivity, 1 ADHD on impulsivity, 2 items on oppositional behavior, 1 item about conduct behavior and 2 items about depressive symptoms. A full description of these items has been previously published (Zwirs et al., 2006).
Impairment measure

The SDQ (Goodman, 1997) comprises 25 items describing positive and negative symptoms divided into 5 scales of 5 items each: Emotional symptoms, Conduct problems, Hyperactivity-Inattention, Peer problems and Social behavior. Each item was scored on a 0-2 scale (not true, somewhat true, certainly true). Additionally, the SDQ includes a short impact supplement that asks about overall distress, social impairment, educational impairment, the burden on the teacher and chronicity. As many teachers knew their pupils for only 6 months, we excluded the item on chronicity. The remaining 4 impact items that are rated on a four-point scale from 0 (not at all) to 3 (very much), were aggregated to generate an impairment score $(\operatorname{Min}=0, \max =12)$. As approximately $80 \%$ of the children scored zero on the impairment score, we decided to dichotomize the score at this value. Accordingly, a score greater than zero was considered as an index of functional impairment.

Analysis

We estimated the population prevalence with $95 \%$ confidence intervals by projecting the observed prevalences in the screen-positive and screen-negative group in the second phase sample to the screen-positive and screen-negative children in the source population, correcting the confidence intervals for the two-stage sampling design (Armitage et al., 1987). We conducted logistic regression to estimate odds ratios $(\mathrm{OR})$ and its $95 \%$ confidence interval $(95 \% \mathrm{CI})$ as measures of the associations of psychiatric disorders with ethnicity, gender, parental psychopathology, peer problems, school problems and the child's country of birth. The analyses with demographic variables (gender and ethnicity) were adjusted for SES. The Dutch children, the girls and the low SES category formed the reference groups. Robust standard errors were used to calculate the confidence intervals to account for clustering by school. Analyses were performed with STATA, version 7.0.

\section{Results}

In Table 1 demographic characteristics of the source population are shown. About half of the children were male and the mean age was almost eight years, which was similar for all ethnic groups. SES was lower among Moroccan and Turkish parents, compared to Dutch and Surinamese parents. The percentage of children that was born in the country of origin was low across all non-Dutch groups, but slightly higher for Surinamese than for Turks and Moroccans. 
Table 1 Demographic characteristics of the study participants

Note. Low SES = Elementary school or no education. The SES distribution regards the distribution in the phase 2 sample.

\begin{tabular}{llllll}
\hline & $\begin{array}{l}\text { Dutch } \\
N=615\end{array}$ & $\begin{array}{l}\text { Moroccan } \\
N=662\end{array}$ & Turkish $N=415$ & $\begin{array}{l}\text { Surinamese } \\
N=349\end{array}$ & $\begin{array}{l}\text { Total } \\
N=2041\end{array}$ \\
\hline $\begin{array}{l}\text { Gender } \\
\quad \text { Boys (\%) }\end{array}$ & 50.1 & 53.5 & 48.7 & 45.0 & 50.0 \\
$\begin{array}{l}\text { Age } \\
\quad \text { Mean (Min; max) }\end{array}$ & $7.6(5 ; 10)$ & $7.9(6 ; 11)$ & $8.0(5 ; 10)$ & $7.7(6 ; 11)$ & $7.8(5 ; 11)$ \\
$\quad \begin{array}{l}\text { SES } \\
\quad \text { Low (\%) }\end{array}$ & 8.9 & 36.9 & 30.9 & 10.3 & 22.0 \\
$\quad \begin{array}{l}\text { Foreign born (\%) } \\
\text { - }\end{array}$ & $67(10.1)$ & $36(8.7)$ & $57(16.3)$ & -
\end{tabular}

Psychiatric disorders and demographic characteristics

Table 2 shows last-year prevalence estimates of DSM-IV diagnoses for the whole sample and stratified by ethnicity and gender. Among Dutch children, 36\% had one or more psychiatric disorders, which was similar for Moroccan $(\mathrm{OR}=1.62 ; 95 \% \mathrm{CI}=0.67-3.91)$, Turkish $(\mathrm{OR}=0.91$; $95 \% \mathrm{CI}=0.39-2.11)$ and Surinamese $(\mathrm{OR}=1.29 ; 95 \%$ $\mathrm{CI}=0.50-3.37$ ) children (Fig. 1). Externalizing Disorders (ED), in particularly ADHD, were more common than mood and anxiety disorders, which was similar for all ethnic groups. The prevalence of externalizing disorders and ADHD among Dutch children was $26 \%$ and $25 \%$, respectively, which did not substantially differ for Moroccan $((\mathrm{OR}=1.51$; $95 \% \mathrm{CI}=0.66-3.46) ;(\mathrm{OR}=1.04 ; 95 \% \mathrm{CI}=0.48-2.23))$, Turkish $((\mathrm{OR}=0.93 ; 95 \% \mathrm{CI}=0.41-2.12) ; \quad(\mathrm{OR}=0.63$; $95 \% \mathrm{CI}=0.26-1.54))$ and Surinamese $((\mathrm{OR}=1.29 ; 95 \%$ $\mathrm{CI}=0.56-2.94) ;(\mathrm{OR}=0.81 ; 95 \% \mathrm{CI}=0.35-1.86))$ children, although Dutch children showed the highest ADHD prevalence.

Age was not related to the prevalence of psychiatric disorders $\left(\mathrm{OR}_{\text {per year }}=0.94 ; 95 \% \mathrm{CI}=0.72-1.22\right)$. Gender effects were generally in the expected direction, i.e. boys showed higher prevalences. In the total group, psychiatric disorders were more frequent in boys than in girls $(\mathrm{OR}=2.87$; $95 \% \mathrm{CI}=1.68-4.90)$, as were externalizing disorders $(\mathrm{OR}=2.71 ; 95 \% \mathrm{CI}=1.66-4.44), \mathrm{ADHD}(\mathrm{OR}=2.53 ; 95 \%$ $\mathrm{CI}=1.38-4.62), \mathrm{CD}(\mathrm{OR}=11.47 ; 95 \% \mathrm{CI}=1.41-92.92)$. No significant difference was observed between boys and girls in the prevalence of ODD $(\mathrm{OR}=1.35 ; 95 \% \mathrm{CI}=0.74$ $2.47)$, anxiety disorders $(\mathrm{OR}=0.60 ; 95 \% \mathrm{CI}=0.20-1.85)$ and mood disorders $(\mathrm{OR}=5.07 ; 95 \% \mathrm{CI}=0.96-26.67)$.

The gender differences observed in the total group were similar for almost all ethnic groups (Fig. 1). Compared to girls, Dutch, and Moroccan boys, had more psychiatric disorders in general ( $(\mathrm{OR}=3.88 ; 95 \% \mathrm{CI}=1.60-9.41)$ and $(\mathrm{OR}=3.87 ; 95 \% \mathrm{CI}=1.37-10.89)$, respectively $)$, and more externalizing disorders $((\mathrm{OR}=4.42 ; 95 \% \mathrm{CI}=1.75-11.13)$ and $(\mathrm{OR}=3.30 ; \quad 95 \% \quad \mathrm{CI}=1.39-7.85)$, respectively). Surinamese boys had more psychiatric disorders in general $(\mathrm{OR}=2.94 ; 95 \% \mathrm{CI}=1.08-8.02)$ when compared to girls. Dutch and Moroccan boys had more ADHD than girls $((\mathrm{OR}=3.96 ; 95 \% \mathrm{CI}=1.52-10.32)$ and $(\mathrm{OR}=2.59$; $95 \% \mathrm{CI}=1.00-6.67)$, respectively). The difference in the

Table 2 One-year prevalence (95\% Confidence Intervals) of DSM-IV diagnostic categories

\begin{tabular}{|c|c|c|c|c|c|c|c|}
\hline & $\begin{array}{l}\text { Any disorder } \\
(95 \% \mathrm{CI})\end{array}$ & $\begin{array}{l}\text { Any ED (95\% } \\
\text { CI) }\end{array}$ & $\begin{array}{l}\text { ADHD } \\
(95 \% \mathrm{CI})\end{array}$ & $\begin{array}{l}\text { ODD } \\
(95 \% \mathrm{CI})\end{array}$ & $\begin{array}{l}\mathrm{CD} \\
(95 \% \mathrm{CI})\end{array}$ & $\begin{array}{l}\text { Any mood }(95 \% \\
\text { CI) }\end{array}$ & $\begin{array}{l}\text { Any anxiety } \\
(95 \% \mathrm{CI})\end{array}$ \\
\hline Total & $33(27-39)$ & $25(19-30)$ & $19(14-24)$ & $11(7-15)$ & $3(1-6)$ & $8(4-11)$ & $8(5-12)$ \\
\hline Boys & $44(35-53)$ & $33(25-41)$ & $25(18-32)$ & $13(7-18)$ & $7(2-11)$ & $12(6-18)$ & $6(2-11)$ \\
\hline Girls & $22(15-30)$ & $16(10-23)$ & $13(7-20)$ & $9(4-14)$ & $0(0-1)$ & $2(0-5)$ & $10(4-16)$ \\
\hline Dutch & $36(23-48)$ & $26(15-37)$ & $25(14-36)$ & $9(2-16)$ & $1(0-2)$ & $6(0-13)$ & $12(4-21)$ \\
\hline Boys & $53(34-72)$ & $40(21-58)$ & $39(21-58)$ & $15(1-28)$ & $2(1-3)$ & $13(0-27)$ & $9(0-20)$ \\
\hline Girls & $21(6-35)$ & $13(2-25)$ & $13(2-25)$ & $5(0-12)$ & 0 & 0 & $15(2-28)$ \\
\hline Moroccan & $38(26-50)$ & $24(14-34)$ & $18(9-26)$ & $8(3-14)$ & $4(0-8)$ & $16(6-26)$ & $10(2-17)$ \\
\hline Boys & $48(31-65)$ & $30(16-44)$ & $20(9-30)$ & $10(2-18)$ & $7(0-15)$ & $22(7-37)$ & $6(0-14)$ \\
\hline Girls & $25(8-42)$ & $16(2-31)$ & $15(1-29)$ & $8(0-17)$ & 0 & $9(0-21)$ & $13(0-27)$ \\
\hline Turkish & $29(17-42)$ & $24(12-36)$ & $16(6-26)$ & $13(4-22)$ & $5(0-11)$ & $3(0-8)$ & $7(0-14)$ \\
\hline Boys & $31(13-49)$ & $25(9-42)$ & $17(3-30)$ & $10(0-21)$ & $9(0-20)$ & $6(0-14)$ & $9(0-20)$ \\
\hline Girls & $28(10-46)$ & $22(5-39)$ & $16(1-32)$ & $16(1-32)$ & 0 & $1(0-2)$ & $5(0-14)$ \\
\hline Surinamese & $30(18-41)$ & $25(15-36)$ & $16(8-25)$ & $13(6-21)$ & $5(0-9)$ & $4(0-9)$ & $3(0-8)$ \\
\hline Boys & $44(26-61)$ & $37(20-53)$ & $25(11-39)$ & $19(7-31)$ & $8(0-17)$ & $8(0-17)$ & 0 \\
\hline Girls & $16(3-28)$ & $15(2-27)$ & $8(0-18)$ & $8(0-18)$ & $1(0-3)$ & 0 & $6(0-16)$ \\
\hline
\end{tabular}

Note. $\mathrm{ED}=$ Externalizing Disorders, i.e. ADHD, ODD and CD; ADHD = Attention-Deficit Hyperactivity Disorder; ODD $=$ Oppositional Defiant Disorder; $\mathrm{CD}=$ Conduct Disorder. 
Fig. Flowchart of sampling procedure and response

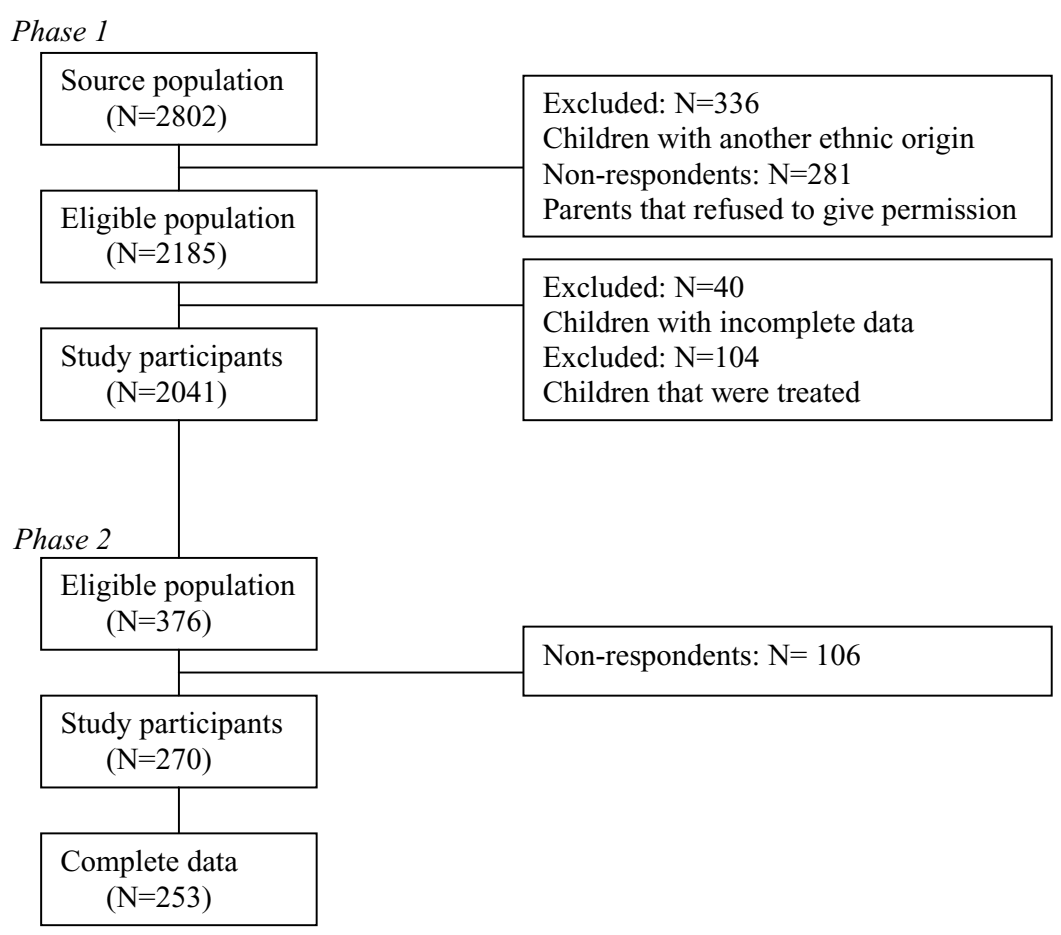

prevalence of externalizing disorders and ADHD between boys and girls was not significant for the Surinamese children $((\mathrm{OR}=2.47 ; 95 \% \mathrm{CI}=0.97-6.30)$ and $(\mathrm{OR}=2.47$; 95\% CI $=0.75-8.12)$, respectively). Among Turkish children, the prevalence of psychiatric disorders did not differ significantly between boys and girls $(\mathrm{OR}=1.16 ; 95 \%$ $\mathrm{CI}=0.36-3.73)$.

Overall, we did not observe an association between SES with the prevalence of any psychiatric disorder, being similar for all ethnic groups $\left(\mathrm{OR}_{\text {high vs low }}=1.24\right.$; $95 \% \mathrm{CI}=0.63-$ 2.43).

In addition, we estimated prevalences that combined bestestimate diagnoses with an impairment measure (Table 3). The overall prevalence dropped from $33 \%$ to $11 \%$, and was similar for Moroccan $(\mathrm{OR}=1.81 ; 95 \% \mathrm{CI}=0.97-3.39)$, Turkish $(\mathrm{OR}=0.98 ; 95 \% \mathrm{CI}=0.41-2.31)$, and Surinamese children $(\mathrm{OR}=0.89 ; 95 \% \mathrm{CI}=0.36-2.17)$, when compared to Dutch children. The proportion children with an impairing externalizing disorder (9\%) was higher than the proportion with a mood $(2 \%)$ or anxiety disorder $(3 \%)$. Among Dutch children, the prevalence of an impairing externalizing disorder or ADHD was $11 \%$, which did not differ for Moroccan $((\mathrm{OR}=1.37 ; 95 \% \mathrm{CI}=0.71-2.67)$ and $(\mathrm{OR}=1.18 ; 95 \%$ $\mathrm{CI}=0.64-2.17))$, Turkish $((\mathrm{OR}=0.77 ; 95 \% \mathrm{CI}=0.32-$ $1.88)$ and $(\mathrm{OR}=0.62 ; 95 \% \mathrm{CI}=0.26-1.51))$ and Surinamese children $((\mathrm{OR}=0.76 ; 95 \% \mathrm{CI}=0.32-1.83)$ and $(\mathrm{OR}=0.62 ; 95 \% \mathrm{CI}=0.24-1.59)$ ).

Gender effects remained in the expected direction. Impairing DSM-IV disorders were more prevalent in boys $\quad(\mathrm{OR}=4.17 ; \quad 95 \% \quad \mathrm{CI}=2.27-7.67), \quad$ as $\quad$ were impairing externalizing disorders $(\mathrm{OR}=5.02 ; 95 \%$ $\mathrm{CI}=2.19-11.50), \quad \mathrm{ADHD} \quad(\mathrm{OR}=4.39 ; 95 \% \mathrm{CI}=1.75-$ 11.01) and ODD (OR=3.06; 95\% $\mathrm{CI}=1.10-8.52)$. The prevalence of impairing $\mathrm{CD}$, mood disorders and
Fig. 1 The overall prevalence of psychiatric disorders separated by ethnicity and gender

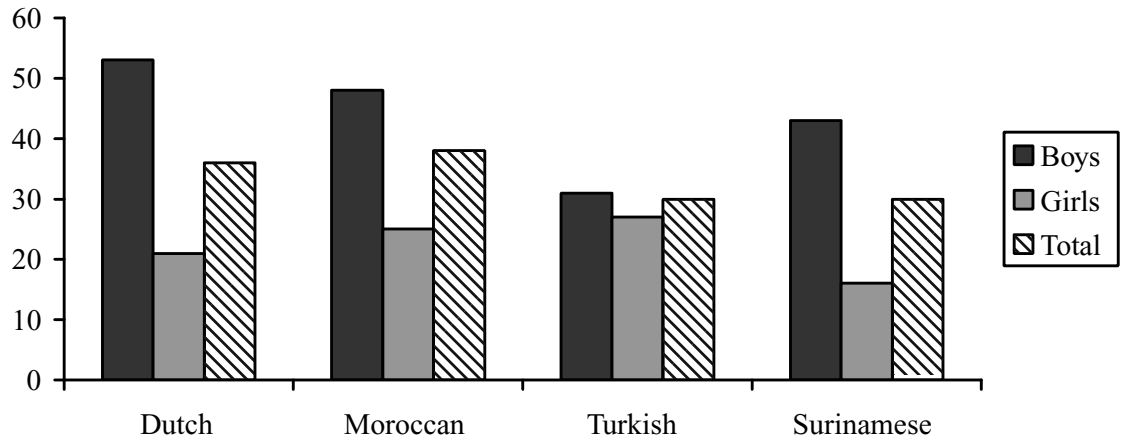


Table 3 One-year prevalence (95\% Confidence Intervals) of DSM-IV diagnostic categories including impairment

\begin{tabular}{|c|c|c|c|c|c|c|c|}
\hline & $\begin{array}{l}\text { Any Disorder } \\
+ \text { impaired } \\
(95 \% \mathrm{CI})\end{array}$ & $\begin{array}{l}\text { Any ED } \\
+ \text { impaired } \\
(95 \% \mathrm{CI})\end{array}$ & $\begin{array}{l}\text { ADHD } \\
+ \text { impaired } \\
(95 \% \mathrm{CI})\end{array}$ & $\begin{array}{l}\text { ODD } \\
+ \text { impaired } \\
(95 \% \mathrm{CI})\end{array}$ & $\begin{array}{l}\text { CD } \\
+ \text { impaired } \\
(95 \% \mathrm{CI})\end{array}$ & $\begin{array}{l}\text { Any Mood } \\
+ \text { impaired } \\
(95 \% \mathrm{CI})\end{array}$ & $\begin{array}{l}\text { Any Anxiety } \\
+ \text { impaired } \\
(95 \% \mathrm{CI})\end{array}$ \\
\hline Total & $11(8-14)$ & $9(6-11)$ & $7(5-10)$ & $4(2-6)$ & $2(1-3)$ & $2(0-3)$ & $3(1-5)$ \\
\hline Boys & $16(11-21)$ & $14(9-19)$ & $12(7-16)$ & $6(3-9)$ & $3(1-5)$ & $2(0-4)$ & $3(0-6)$ \\
\hline Girls & $5(2-9)$ & $3(0-5)$ & $2(0-5)$ & $2(0-4)$ & $0(0-1)$ & $1(0-4)$ & $2(0-5)$ \\
\hline Dutch & $11(4-18)$ & $11(4-18)$ & $11(3-18)$ & $5(0-10)$ & $1(0-2)$ & $0(0-1)$ & $0(0-1)$ \\
\hline Boys & $22(7-37)$ & $22(7-37)$ & $22(7-37)$ & $10(0-21)$ & $2(1-3)$ & $1(0-1)$ & $1(0-1)$ \\
\hline Girls & $1(0-3)$ & $1(0-3)$ & $1(0-3)$ & $0(0-0)$ & $0(0-0)$ & $0(0-0)$ & $0(0-0)$ \\
\hline Moroccan & $13(7-19)$ & $8(6-10)$ & $6(4-8)$ & $4(2-5)$ & $2(1-3)$ & $5(0-11)$ & $5(0-11)$ \\
\hline Boys & $18(10-26)$ & $12(10-15)$ & $10(7-13)$ & $5(3-8)$ & $3(1-5)$ & $5(0-13)$ & $6(0-14)$ \\
\hline Girls & $6(0-15)$ & $2(0-4)$ & $2(0-4)$ & $2(0-4)$ & $0(0-0)$ & $4(0-13)$ & $4(0-13)$ \\
\hline Turkish & $12(4-21)$ & $9(2-17)$ & $6(0-13)$ & $4(0-8)$ & $2(0-7)$ & $0(0-1)$ & $5(0-11)$ \\
\hline Boys & $11(0-23)$ & $11(0-23)$ & $7(0-15)$ & $1(0-3)$ & $4(0-13)$ & $0(0-0)$ & $1(0-3)$ \\
\hline Girls & $13(0-26)$ & $7(0-17)$ & $6(0-16)$ & $6(0-16)$ & $0(0-0)$ & $1(0-2)$ & $5(0-14)$ \\
\hline Surinamese & $6(5-8)$ & $6(4-7)$ & $5(3-6)$ & $4(2-6)$ & $2(0-3)$ & $1(0-2)$ & $0(0-0)$ \\
\hline Boys & $12(9-14)$ & $11(8-13)$ & $8(5-11)$ & $8(5-11)$ & $2(0-4)$ & $2(0-4)$ & $0(0-0)$ \\
\hline Girls & $1(0-3)$ & $1(0-3)$ & $1(0-3)$ & $0(0-0)$ & $1(0-3)$ & $0(0-0)$ & $0(0-0)$ \\
\hline
\end{tabular}

Note. $\mathrm{ED}=$ Externalizing Disorders, i.e. ADHD, ODD and CD; ADHD = Attention-Deficit Hyperactivity Disorder; ODD = Oppositional Defiant Disorder; $\mathrm{CD}=$ Conduct Disorder.

anxiety disorders was not significantly higher for boys $((\mathrm{OR}=8.25 ; 95 \% \quad \mathrm{CI}=0.97-69.82), \quad(\mathrm{OR}=2.20 ; 95 \%$ $\mathrm{CI}=0.61-7.90)$ and $(\mathrm{OR}=2.15 ; 95 \% \mathrm{CI}=0.39-11.98)$, respectively).

The pattern of gender effects observed in the total group was similar to the pattern observed in almost all separate ethnic groups. The prevalence of any impairing DSM-IV disorder and impairing externalizing disorder was higher in Dutch $((\mathrm{OR}=14.60 ; 95 \% \mathrm{CI}=1.66-128.87)$ and $(\mathrm{OR}=14.60$; $95 \% \mathrm{CI}=1.66-128.87))$ and Moroccan boys $((\mathrm{OR}=6.12$; $95 \% \mathrm{CI}=2.26-16.56)$ and $(\mathrm{OR}=6.94 ; 95 \% \mathrm{CI}=1.45-$ 33.23)), when compared to girls. Dutch boys had more impairing $\mathrm{ADHD}(\mathrm{OR}=12.95 ; 95 \% \mathrm{CI}=1.55-107.81)$ than girls. The prevalence of ADHD was non-significantly higher for Moroccan boys than for girls $(\mathrm{OR}=4.79$; $95 \%$ $\mathrm{CI}=0.99-23.07)$. The prevalence of any impairing DSMIV disorder, externalizing disorders and ADHD was nonsignificantly higher in Surinamese boys $((\mathrm{OR}=8.55 ; 95 \%$ $\mathrm{CI}=0.80-90.99),(\mathrm{OR}=7.24 ; 95 \% \mathrm{CI}=0.73-72.13)$ and ( $\mathrm{OR}=4.96$; 95\% CI $=0.48-51.39)$, respectively), as compared to girls. Among Turkish children, the prevalence for any impairing DSM-IV diagnosis, impairing externalizing disorder or ADHD did not differ between boys and girls $((\mathrm{OR}=0.59 ; 95 \% \mathrm{CI}=0.16-2.14),(\mathrm{OR}=0.92$; $95 \% \mathrm{CI}=0.22-3.77)$ and $(\mathrm{OR}=0.96 ; 95 \% \mathrm{CI}=0.15-6.06)$, respectively).

Again, we observed no relation between SES and any impairing psychiatric disorder, which was similar for all ethnic groups $(\mathrm{OR}=0.89 ; 95 \% \mathrm{CI}=0.49-1.61)$.
Psychiatric disorders and psychosocial mechanisms

In the total group, we observed a positive relationship between the prevalence of psychiatric disorders among children and psychopathology in their parents $(\mathrm{OR}=3.79$; $95 \% \mathrm{CI}=1.55-9.30)$. This association was similar for the Dutch $(\mathrm{OR}=9.24 ; 95 \% \mathrm{CI}=1.96-43.62)$ and the Turkish $(\mathrm{OR}=5.71 ; 95 \% \mathrm{CI}=1.57-20.84)$, but not for the Moroccan $(\mathrm{OR}=2.84 ; 95 \% \mathrm{CI}=0.47-17.30)$, and the Surinamese $(\mathrm{OR}=0.75 ; 95 \% \mathrm{CI}=0.10-5.50)$ group.

A positive association was observed between the prevalence of psychiatric disorders and peer problems $(\mathrm{OR}=1.31$; $95 \% \mathrm{CI}=1.15-1.50)$ in the total group. The relationship with peer problems as observed in the total group was similar for the Moroccan $(\mathrm{OR}=1.32 ; 95 \% \mathrm{CI}=1.03-1.69)$, the Turkish $(\mathrm{OR}=1.69 ; 95 \% \mathrm{CI}=1.25-2.28)$ and the Surinamese group $(\mathrm{OR}=1.26 ; 95 \% \mathrm{CI}=1.00-1.57)$, but not for the Dutch $(\mathrm{OR}=1.14 ; 95 \% \mathrm{CI}=0.92-1.42)$.

We observed a positive association between the prevalence of psychiatric disorders and school problems $(\mathrm{OR}=1.44 ; 95 \% \mathrm{CI}=1.28-1.62)$. This relationship was similar for the Dutch $(\mathrm{OR}=1.45 ; 95 \% \mathrm{CI}=1.17$ 1.78), Moroccan $(\mathrm{OR}=1.41 ; 95 \% \mathrm{CI}=1.13-1.77)$, Turkish $(\mathrm{OR}=1.38 ; 95 \% \mathrm{CI}=1.14-1.68)$ and Surinamese group $(\mathrm{OR}=1.57 ; 95 \% \mathrm{CI}=1.21-2.04)$.

Among the non-Dutch groups, no association was observed between the prevalence of psychiatric disorders and the child's country of birth $(p=0.87)$, mother's country of birth $(p=0.87)$ and father's country of birth $(p=0.32)$. 
The above analyses of psychosocial mediators were not adjusted for SES as this factor was not associated with psychiatric disorders in children.

\section{Discussion}

The present study is the first epidemiological study in Europe that estimated the prevalence of childhood psychiatric disorders and associated mechanisms based on best-estimate diagnoses in four different ethnic groups from one population. It appeared that the prevalence of psychiatric disorders did not differ between native Dutch and immigrant nontreated children. Generally, psychiatric disorders were more prevalent among boys than among girls with the exception of ODD, anxiety disorders and mood disorders which did not differ significantly across gender.

The overall prevalence of psychiatric disorders (including the impairment measure) was estimated at $11 \%$, among which externalizing disorders $(9 \%)$ were more common than mood disorders $(2 \%)$ and anxiety disorders $(3 \%)$. This prevalence estimate is comparable to the prevalence observed in another study conducted in the Netherlands (Verhulst, van der, Ferdinand, \& Kasius, 1997) and to the prevalence estimates in other countries (Ford, Goodman, \& Meltzer, 2003; Bilenberg, Petersen, Hoerder, \& Gillberg, 2005).

Contrary to our expectation, the prevalence of psychiatric disorders was similar for Western native and non-Western immigrant children. Possibly, the influence of living in a low SES inner-city neighbourhood overshadows ethnic effects. Moreover, ethnic differences in the prevalence of psychiatric disorders may not exist in childhood, but may emerge as children grow older, for instance because of the increasing impact of discrimination. This finding of similar prevalence rates across ethnicity is consistent with the results of comparable studies in the US (Costello et al., 1997; Angold et al., 2002), but in contrast to other studies (Bird, 1996; Crijnen, Achenbach, \& Verhulst, 1997; Roberts et al., 1998). However, the latter studies compared prevalence estimates in different populations using different methodologies which, at least in part, may explain the observed differences.

The few European studies that compared native and immigrant children offered contradictory results regarding the ethnic differences in the level of problem behavior, depending on which informant provided the data. For instance, Moroccan immigrant adolescents themselves reported fewer problems than their Dutch native counterparts, whereas parents observed no difference in the level of problem behavior, in contrast to teachers who reported more externalizing problems for Moroccan than for native Dutch youth (Stevens et al., 2003). Likewise, according to teacher ratings Turkish immigrant and native Dutch children display similar levels of problem behavior (Crijnen et al., 2000), whereas according to self-reports the level of problem behavior differed between
Turkish immigrant and Dutch children (Murad et al., 2003). Nevertheless, these studies relied on rating scales and lacked a gold standard. As a result, the observed differences in previous studies may be due to perceptual differences rather than to actual differences in problem behavior.

The second goal was to get insight into the associated mechanisms of psychiatric disorders in Dutch, Moroccan, Turkish and Surinamese children. Overall, our findings are consistent with results from previous studies. No association was observed between the prevalence of psychiatric disorders and age which may be explained by the restricted age range of the sample (6-10 years). Gender differences in the prevalence of psychiatric disorders appeared to be crossculturally invariant, as boys had more psychiatric disorders than girls in all ethnic groups, except for the Turks, accounted for largely by a relatively lower prevalence among Turkish boys. This cross-cultural consistency in gender effects is in accordance with previous findings (Crijnen, Achenbach, \& Verhulst, 1999). The relatively low prevalence of psychiatric disorders among Turkish boys is in line with a study in which Turkish boys reported less delinquent behavior than Dutch boys (Murad et al., 2003). Possibly, the internal social cohesion and social control which has found to be stronger in Turkish culture than in other cultures (Dagevos, 2001; Pels, 2003), may be an important mechanism in protecting Turkish boys from developing externalizing disorders.

In contrast with previous results (Verhulst \& Achenbach, 1995a), we observed no association between the prevalence of psychiatric disorders and SES, i.e. parents' highest educational level. Possibly, the variation in SES in these low SES inner-city areas is too small to detect associations between SES and the prevalence of psychiatric disorders. Another explanation may be that the influence of living in a deprived neighborhood outweighs the effect of the individual level of SES on the prevalence of psychiatric disorders (Kalff et al., 2001).

Consistent with previous results (Johnson et al., 2006), parental psychopathology was related to psychiatric disorders in the total group. This association was also observed in the Dutch and Turkish group, but not in the other ethnic groups. This is in line with a study among Moroccan adolescents in which parental psychopathology was hardly related to externalizing problems (Stevens, Vollebergh, Pels, \& Crijnen, 2005a), neither to internalizing problems (Stevens, Vollebergh, Pels, \& Crijnen, 2005b). Taking into account the finding that peer problems were related to psychiatric disorders among Turkish and Moroccan children, but not among Dutch and Surinamese children, it may be argued that among non-Dutch immigrant children peer factors are stronger related to psychiatric disorders than parental factors.

In accordance with previous findings (Sowa et al., 2000; Stevens et al., 2005a; b), school problems were positively related to psychiatric disorders among all ethnic groups. 
Among the non-Dutch groups no effect was observed from the child's country of birth, mother's country of birth or father's country of birth. This may be explained by the fact that most children (89\%) were born in the Netherlands and most parents were born in the country of origin (97\%). Other migration factors, such as discrimination or acculturation problems (Stevens et al., 2005a,b) may be better predictors of psychiatric disorders.

The results of this study are subject to some limitations. First, the members of the diagnostic panel in our study were all of Dutch origin, and may therefore be biased in assessing non-Dutch children. For instance, Sonuga-Barke et al. (1993) reported that English teachers rated Asian immigrant children higher on hyperactivity symptoms than English native children, whereas the scores on more objective measures of hyperactivity were similarly for both groups (Sonuga-Barke $\&$ Minocha, 1993). Nevertheless, the members of our diagnostic panel and the expert panel were qualified in working with non-Dutch children, in that they received specialized training in working with non-Dutch youth and were competent and proficient in the worldview and cultures represented by study participants. Therefore, we consider the possibility of perceptual bias of limited size. Second, the DISC-P had not been validated in a Moroccan, Turkish or Surinamese sample. However, as we conducted a pilot study among Moroccan and Turkish parents, and as our translations result from consultation of different experts and from extensive discussions among the carefully trained bicultural/bilingual interviewers, we consider our translations as relatively valid. Moreover, it would probably be impossible to develop a 'standard translation' for Moroccans as Berber (the mother tongue of $70 \%$ of the Moroccans in the Netherlands) is an oral language of which many variations do exist. Still, it is of great importance that instruments like the DISC-P will be validated in non-Western samples like Moroccans, Turks and Surinamese to illuminate the meaning of specific items for Moroccans, Turks and Surinamese and to find out whether these ethnic groups hold different concepts of externalizing disorders. Third, as only $160(11.2 \%)$ of the non-Dutch children in our study were born in the country of origin and as we have sampled from disadvantaged inner-city neighborhoods, our results concern second generation immigrants from low SES urban areas, and may not generalize to first generation immigrant children or to neighborhoods with middle and higher SES levels without additional study. Nevertheless, as most migrants are from the lowest SES, generalizing to middle and higher SES neighborhoods seems of less relevance. Fourth, in comparison with other epidemiological studies, the sample size in the second stage of the present study was rather small, which reduces the statistical power to detect small differences. Yet, the stability of the point estimates of the prevalence across gender and ethnicity groups suggests that this problem is limited. Finally, using a cross-sectional design, it was not possible to establish the causal direction of the observed associations. For instance, it is as likely that peer problems lead to psychiatric disorders as that psychiatric disorders result in peer problems.

Implications and future research

In conclusion, our study shows that the prevalence of childhood disorders does not differ for untreated Western native and non-Western immigrant children in low SES innercity neighborhoods. As we studied different ethnic groups from one population using one single methodology including uniform diagnostic assessments, our findings may indicate that previously observed differences in prevalence estimates (Bird, 1996; Roberts et al., 1998; Stevens et al., 2003; Murad et al., 2003) may at least in part be due to methodological or SES variations. Therefore, it is of great importance to study the effect of ethnicity on the prevalence of psychiatric disorders by comparing ethnic groups from one population using one single methodology and best-estimate diagnoses.

Our finding that the prevalence of psychiatric disorders is similar for immigrant children and their native counterparts who reside in the same low SES inner-city neighborhoods, has not only relevance for the Netherlands, but also for other multiethnic societies, as immigrants in these countries are also highly concentrated in poor urban neighborhoods. As a result, the prevalence estimates in low SES urban areas in these countries may parallel the currently observed prevalence estimates. However, although the prevalence of psychiatric disorders in low SES inner-city areas may be similar for native and immigrant children, the prevalence and the effects of risk and protective factors may differ according to ethnicity as indicated by the present findings and previous studies (Deater-Deckard \& Dodge, 1997; Beiser, Hou, Hyman, \& Tousignant, 2002).

Acknowledgments This study was financially supported by The Netherlands Organization for Scientific Research (ZON-MW), grant number 99-9.1-64, by the Foundation for Children's Welfare Stamps Netherlands and by the Province of Utrecht, the Netherlands.

Declaration of interest Dr Buitelaar has served as consultant and has been on advisory boards for Eli Lilly, Janssen Cilag BV, UCB, and Shire in the past two years. The other authors have no financial relationships to disclose.

\section{References}

Diagnostic and Statistical Manual of Mental Disorders-Fourth edition DSM-IV (1994). Washington, DC: American Psychiatric Association.

Angold, A., Erkanli, A., Farmer, E. M., Fairbank, J. A., Burns, B. J., Keeler, G., et al. (2002). Psychiatric disorder, impairment, and service use in rural African American and white youth. Archives of General Psychiatry, 59, 893-901. 
Armitage, P., Berry, G., \& Matthews, J. N. S. (1987). Statistical Methods in Medical Research (Second ed.). Oxford: Blackwell Scientific Publications.

Beiser, M., Hou, F., Hyman, I., \& Tousignant, M. (2002). Poverty, family process, and the mental health of immigrant children in Canada. American Journal of Public Health, 92, 220-227.

Bengi-Arslan, L., Verhulst, F. C., Van Der Ende, J., \& Erol, N. (1997). Understanding childhood (problem) behaviors from a cultural perspective: comparison of problem behaviors and competencies in Turkish immigrant, turkish and Dutch children. Social Psychiatry and Psychiatr Epidemiology, 32, 477-484.

Bilenberg, N., Petersen, D. J., Hoerder, K., \& Gillberg, C. (2005). The prevalence of child-psychiatric disorders among 8-9-year-old children in Danish mainstream schools. Acta Psychiatrica Scandinavica, 111, 59-67.

Bird, H. R. (1996). Epidemiology of childhood disorders in a crosscultural context. Journal of Child Psychology Psychiatry, 37, $35-49$.

Bird, H. R., Canino, G., Rubio-Stipec, M., Gould, M. S., Ribera, J., Sesman, M. et al. (1988). Estimates of the prevalence of childhood maladjustment in a community survey in Puerto Rico. The use of combined measures. Archives of General Psychiatry, $45,1120-1126$.

Canino, G., Shrout, P. E., Rubio-Stipec, M., Bird, H. R., Bravo, M., Ramirez, R., et al. (2004). The DSM-IV rates of child and adolescent disorders in Puerto Rico: Prevalence, correlates, service use, and the effects of impairment. Archives of General Psychiatry, 61, 85-93.

Costello, E. J., Farmer, E. M., Angold, A., Burns, B. J., \& Erkanli, A. (1997). Psychiatric disorders among American Indian and white youth in Appalachia: the Great Smoky Mountains Study. American Journal of Public Health, 87, 827-832.

Crijnen, A. A., Achenbach, T. M., \& Verhulst, F. C. (1999). Problems reported by parents of children in multiple cultures: the Child Behavior Checklist syndrome constructs. American Journal of Psychiatry, 156, 569-574.

Crijnen, A. A., Bengi-Arslan, L., \& Verhulst, F. C. (2000). Teacherreported problem behaviour in Turkish immigrant and Dutch children: a cross-cultural comparison. Acta Psychiatrica Scandinavica, 102, 439-444.

Crijnen, A. A. M., Achenbach, T. M., \& Verhulst, F. C. (1997). Comparisons of problems reported by parents of children in 12 cultures: Total problems, externalizing and internalizing. Journal of the American Academy of Child and Adolescent Psychiatry, $36,1269-1277$.

Dagevos, J. (2001). Perspectief op integratie; Over de sociaal-culturele en structurele integratie van etnische minderheden in Nederland (Perspective on integration; On the social-cultural position of ethnic minorities in the Netherlands). Den Haag: Wetenschappelijke raad voor het regeringsbeleid.

Deater-Deckard, K., \& Dodge, K. A. (1997). Externalizing behavior problems and discipline revisited: Nonlinear effects and variation by culture, context, and gender. US: Lawrence Erlbaum.

Ford, T., Goodman, R., \& Meltzer, H. (2003). The British Child and Adolescent Mental Health Survey 1999: the prevalence of DSM-IV disorders. Journal of the American Academy of Child and Adolescent Psychiatry, 42, 1203-1211.

Goodman, R. (1997). The strengths and difficulties questionnaire: a research note. Journal of Child Psychology and Psychiatry, 38, $581-586$.

Johnson, J. G., Cohen, P., Kasen, S., \& Brook, J. S. (2006). A multiwave inulti-informant study of the specificity of the association between parental and offspring psychiatric disorders. Comprehensive Psychiatry, 47, 169-177.

Kalff, A. C., Kroes, M., Vles, J. S., Hendriksen, J. G., Feron, F. J., Steyaert, J., et al. (2001). Neighbourhood level and individual level SES effects on child problem behaviour: a multilevel analysis. Journal of Epidemiology Community Health, 55, 246250.

Kashani, J. H., Beck, N. C., Hoeper, E. W., Fallahi, C., Corcoran, C. M., McAllister, J. A., et al. (1987). Psychiatric disorders in a community sample of adolescents. American Journal of Psychiatry, 144, 584-589.

Kasius, M. C. (1997). Interviewing children. Development of the Dutch version of the Semi-structured Clinical Interview for Children and Adolescents (SCICA) and testing of the psychometric properties. Rotterdam: Unpublished doctoral dissertation, Erasmus University, The Netherlands.

Knol, F. A. (1998). Van hoog naar laag; van laag naar hoog (From high to low; from low to high). Den Haag: Sociaal en Cultureel Planbureau.

Kosten, T. A., \& Rounsaville, B. J. (1992). Sensitivity of psychiatric diagnosis based on the best estimate procedure. American Journal of Psychiatry, 149, 1225-1227.

Kvernmo, S., \& Heyerdahl, S. (1998). Influence of ethnic factors on behavior problems in indigenous Sami and majority Norwegian adolescents. Journal of the American Academy of Child and Adolescent Psychiatry, 37, 743-751.

McConaughy, S. H., \& Achenbach, T. M. (2001). Manual for the Semistructured Clinical Interview for Children and Adolescents. Burlington: University of Vermont.

Murad, S. D., Joung, I. M., van Lenthe, F. J., Bengi-Arslan, L., \& Crijnen, A. A. (2003). Predictors of self-reported problem behaviours in Turkish immigrant and Dutch adolescents in the Netherlands. Journal of Child Psychology Psychiatry, 44, $412-423$.

Pels, T. (2003). Respect van twee kanten; Een studie over last van Marokkaanse jongeren. Assen: Koninklijke van Gorcum.

Roberts, R. E., Attkisson, C. C., \& Rosenblatt, A. (1998). Prevalence of psychopathology among children and adolescents. American Journal of Psychiatry, 155, 715-725.

Schwab-Stone, M. E., Shaffer, D., Dulcan, M. K., Jensen, P. S., Fisher, P., Bird, H. R., et al. (1996). Criterion validity of the NIMH Diagnostic Interview Schedule for Children Version 2.3 (DISC-2.3). Journal of American Academy Child Adolescent Psychiatry, 35, 878-888.

Shaffer, D., Fisher, P., Lucas, C. P., Duldan, M. K., \& Schwab-Stone, M. E. (2000). NIMH Diagnostic Interview Schedule for Children Version IV (NIMH DISC-IV): Description, differences from previous versions, and reliability of some common diagnoses. Journal of American Academy Child Adolescent Psychiatry, 39, 28-38.

Sonuga-Barke, E. J. S., \& Minocha, K. (1993). Inter-ethnic bias in teachers' ratings of childhood hyperactivity. British Journal of Developmental Psychology, 11, 187-200.

Sowa, H., Crijnen, A. A., Bengi-Arslan, L., \& Verhulst, F. C. (2000). Factors associated with problem behaviors in Turkish immigrant children in The Netherlands. Social Psychiatry and Psychiatr Epidemiology, 35, 177-184.

Stevens, G. W., Pels, T., Bengi-Arslan, L., Verhulst, F. C., Vollebergh, W. A., \& Crijnen, A. A. (2003). Parent, teacher and self-reported problem behavior in The Netherlands: comparing Moroccan immigrant with Dutch and with Turkish immigrant children and adolescents. Social Psychiatry and Psychiatr Epidemiology, 38, $576-585$.

Stevens, G. W. J. M., Vollebergh, W. A. M., Pels, T. V. M., \& Crijnen, A. A. M. (2005a). Predicting externalizing problems in Moroccan immigrant adolescents in the Netherlands. Social Psychiatry and Psychiatric Epidemiology, 40, 571-579.

Stevens, G. W. J. M., Vollebergh, W. A. M., Pels, T. V. M., \& Crijnen, A. A. M. (2005b). Predicting internalizing problems in Moroccan immigrant adolescents in the Netherlands. Social Psychiatry and Psychiatric Epidemiology, 40, 1003-1011. 
Verhulst, F. C., \& Achenbach, T. M. (1995b). Empirically based assessment and taxonomy of psychopathology: cross-cultural applications. A review. European Child and Adolescent Psychiatry, 4, 61-76.

Verhulst, F. C., \& Achenbach, T. M. (1995a). Empirically based assessment and taxonomy of psychopathology: cross-cultural applications. A review. European Child and Adolescent Psychiatry, 4, 61-76.

Verhulst, F. C., van der, E. J., Ferdinand, R. F., \& Kasius, M. C. (1997). The prevalence of DSM-III-R diagnoses in a national sample of Dutch adolescents. Archives General Psychiatry, 54, 329336

Zwirs, B. W. C., Burger, H., Schulpen, T. W. J., \& Buitelaar, J. K. (2006). Different treatment thresholds in non-western children with behavioral problems. Journal of the American Academy of Child and Adolescent Psychiatry, 45, 476-483.

Zwirs, B. W. C., Burger, H., Buitelaar, J. K., \& Schulpen, T. W. J. (2006). Ethnic differences in parental detection of externalizing disorders. European Child \& Adolescent Psychiatry, 15, 418426. 